Original Research

\title{
Prediction of one- and two-year mortality after transcatheter aortic valve implantation: proposal of a fast sum-score system integrating a novel biomarker of cardiac extracellular matrix accumulation and fibrosis
}

\author{
Laura Bäz ${ }^{1}$, Katja Grün ${ }^{1}$, Mahmoud Diab ${ }^{2}$, Alexander Pfeil ${ }^{3}$, Christian Jung ${ }^{4}$, \\ Sven Möbius-Winkler ${ }^{1}$, P. Christian Schulze ${ }^{1}$, Marcus Franz ${ }^{1, *}$ \\ ${ }^{1}$ Department of Internal Medicine I, University Hospital Jena, Friedrich-Schiller-University Jena, 07740 Jena, Germany \\ ${ }^{2}$ Department of Cardiothoracic Surgery, University Hospital Jena, Friedrich-Schiller-University Jena, 07740 Jena, Germany \\ ${ }^{3}$ Department of Internal Medicine III, University Hospital Jena, Friedrich-Schiller-University Jena, 07740 Jena, Germany \\ ${ }^{4}$ Division of Cardiology, Pulmonology and Vascular Medicine, Medical Faculty, University of Düsseldorf, 40225 Düsseldorf, Germany \\ *Correspondence: Marcus.Franz@med.uni-jena.de (Marcus Franz) \\ Academic Editor: Giuseppe Santarpino \\ Submitted: 22 November 2021 Revised: 4 January 2022 Accepted: 11 January 2022 Published: 14 February 2022
}

\begin{abstract}
Background: Prediction of long-term mortality in patients with severe symptomatic aortic valve stenosis undergoing transcatheter aortic valve implantation (TAVI) is still challenging but of great impact with respect to the selection of treatment strategy. Whereas most of the established scores address perioperative risk and/or short-term mortality, the aim of our current study was the integrative investigation of a multitude of patients' characteristics including novel biomarkers of cardiovascular remodeling with respect to their value for the prediction of long-term mortality. Methods: In a first subset of patients $(n=122$, identification group) a wide range of baseline characteristics were assigned to three clusters with 4 to 10 items each (classical clinical parameters; risk assessment scores; novel biomarkers of cardiovascular remodeling) and tested with respect to their predictive value for one-year mortality. Thereby, a sum-score system (Jena Mortality Score, JMS) was defined and tested in a larger collective of TAVI patients $(n=295$, validation group $)$ with respect to one- and two-year mortality prediction. Results: In the identification cohort, binary logistic regression analysis, with one-year mortality as dependent variable and the items per cluster as cofounders, revealed atrial fibrillation (Afib; odds ratio [OR] 7.583, 95\% confidence interval [95\% CI]: 2.051-28.040, $p=0.002$ ), clinical frailty scale (CFS; OR 2.258, 95\% CI: 1.262-4.039, $p=0.006$ ) and Tissue-Inhibitor of Metalloproeinase-1 (TIMP-1; OR $1.006,95 \%$ CI: $1.001-1.011, p=0.019)$ as independent predictors of one-year mortality. These 3 parameters were integrated into a simplified sum-score as follows: presence of Afib (no $=0$, yes $=1)$; dichotomized CFS $(1$ to $4=0 ; 5$ to $9=1)$; TIMP- 1 range (cut-off value $187.2 \mathrm{ng} / \mathrm{mL}$; below $=0$, above $=1$ ). The resulting sum-score (JMS) ranged from 0 to 3 . By binary logistic regression analysis in the validation cohort with one- and two-year mortality as dependent variable and Society of Thoracic Surgeons (STS) score (STS), staging of extra-valvular cardiac damage (stage), presence of high gradient aortic stenosis (HGAS), EQ visual analogue scale score (EQ-VAS) and JMS as cofounders, besides STS score, only JMS could be proven to serve as independent predictor of both, one-year (OR 1.684, 95\% CI: $1.094-2.592, p=0.018$ ) and two-year (OR 1.711, 95\% CI: 1.136-2.576, $p=0.010$ ) mortality. After dichotomization of patients into a low-risk and a high-risk group according to JMS, Kaplan-Meier survival analysis displayed a significant survival benefit for the low-risk group after one and two years $(p<0.001)$. Conclusion: JMS, including TIMP-1 as a novel biomarker of cardiac extracellular matrix accumulation and fibrosis, could serve as a novel simple tool to assess long-term mortality risk after TAVI and might thereby contribute to a more precise stratification of individual risk.
\end{abstract}

Keywords: TAVI; risk prediction; mortality; biomarker; TIMP-1

\section{Introduction}

Transcatheter aortic valve implantation (TAVI) as an alternative for surgical aortic valve replacement (SAVR) for the treatment of severe symptomatic aortic valve stenosis (AS) has rapidly developed in the last decade and represents the treatment of choice in patients of 75 years or older, irrespective of surgical risk [1-5]. Prediction of long-term mortality in patients undergoing TAVI is still challenging but of great impact with respect to the selection of the appropriate treatment strategy (SAVR versus TAVI), especially in the low- or moderate risk group between 70 and 75 years of age. Current guidelines and consensus papers claim the evaluation of these patients in the frame of a heart team to recommend the optimal treatment for each individual patient $[6,7]$. Thus, the availability of risk scores, which are at once reliable and easy to perform in daily practice, are of great clinical interest. Currently, the majority of risk scores used for TAVI patients are borrowed from surgical mortality prediction tools, which are known to overestimate short-term mortality in TAVI patients, especially in the elective set- 
ting [8-10]. The most commonly used scores are EuroScore II and the Society of Thoracic Surgeons Predicted Risk of Mortality model (STS-PROM) [6,11,12]. Motivated by the above-mentioned limitations of the surgical scores, a variety of TAVI specific mortality prediction models have been proposed in the last years, e.g., the survival post TAVI score (STT), the OBSERVANT score or the FRANCE-2 score [13-15]. A common problem of these scores is the fact that they were mainly derived from the very early TAVI collectives, which were characterized by an extremely high (prohibitive) or high surgical risk. Thus, their transferability to present-day TAVI patients, which summarize elderly patients of all surgical risk categories, is very limited and the scores have not performed satisfactory in external validation studies [10].

Another unmet clinical need, in particular when talking about moderate or low surgical risk patients, is the missing appropriateness of TAVI specific scores to predict longterm mortality reflecting patients' survival beyond the procedure. Thus, reliable prediction of one- or even two-year mortality would be of great interest for the individual patient as well as the health care system $[10,16,17]$.

To reach the goal of defining a risk prediction model fulfilling all these requirements, one has to expand conventional scoring parameters by the integrative implementation of further prognostic determinants derived from, e.g., individual frailty assessment, imaging modalities or circulating reflectors of cardiovascular tissue remodeling. Especially the latter aspect was largely neglected in the past causing that those novel biomarkers, which have been shown to be prognostic in representative cohorts, are not implemented in TAVI risk prediction tools yet [10,18-27].

The aim of our current preliminary study was the integrative investigation of a multitude of patients' characteristics including frailty assessment and novel biomarkers of cardiovascular remodeling with respect to their value for the prediction of long-term mortality in real-life patients undergoing TAVI.

\section{Material and methods}

\subsection{Patient cohorts}

The Jenaer Aortenklappenregister (JAKR), established in 2016, prospectively includes all patients that undergo transcatheter aortic valve implantation (TAVI) at the University Hospital Jena. In this study, we investigated two patient cohorts with the aim to, first, identify novel markers for mortality risk prediction and define a simple sum-score system (identification cohort, $\mathrm{n}=122$ ) and second, validate it in a larger study population (validation cohort, $\mathrm{n}=295$ ). All patients included gave written informed consent for participation and the local ethics committee of the University Hospital Jena has approved the study (registration number: 4815-06/16). Detailed clinical, laboratory, functional and imaging analysis according to local standard operating procedures were performed in adherence to the principles of the current version of the Declaration of Helsinki and good clinical practice guidelines. Structured patient follow-up was carried out at 6 weeks, 6 months, 12 months and 24 months according to the JAKR protocol. In principle, all patients undergoing TAVI and agreed to participate were consecutively included in the study. Due to potential extracardiovascular sources of the serum biomarkers determined by Enzyme linked Immunosorbent Assay (ELISA), the following conditions were excluded: active malignant or autoimmune disease, hyperthyroidism, infections or systemic intake of corticosteroids.

\subsection{Acquisition of health status and clinical frailty}

For the assessment of patients' health status, the EuroQol visual analogue scale (EQ-VAS; between 0 and 100 points) was used as a component of the EuroQol questionnaire (EQ-5D-5L), which is an accepted tool for the standardized simple generic Quality of Life (QoL) assessment already used in TAVI patients [28-30]. The Clinical Frailty Scale (CFS), known to predict death or the need for institutional care, was applied to assess clinical frailty of the patients by assigning the following categories: very fit (1), well (2), managing well (3), vulnerable (4), mildly frail (5), moderately frail (6), severely frail (7), very severely frail (8) and terminally ill (9) [31].

\subsection{Blood sample acquisition and measurement of routine laboratory parameters as well as quantification of serum levels of novel biomarkers of cardiovascular remodelling}

In the frame of the JAKR study protocol, blood samples were taken from all study participants by standard venous puncture. The collection tubes were centrifuged within $20 \mathrm{~min}$ after withdrawal and serum was transferred into special low binding tubes (Protein LoBind, Eppendorf AG, Hamburg, Germany), snap frozen in liquid nitrogen to reduce artificial protein degradation and finally stored at $-80{ }^{\circ} \mathrm{C}$ until further analysis. Routine laboratory parameters, e.g., brain natriuretic peptide (BNP) levels or creatinine, were measured according to local standard operating procedures. The circulating levels of preselected biomarkers of cardiovascular tissue remodelling were quantified by ELISA technique. For the following biomarkers, commercially available assays were used according to the instructions of the manufacturer: matrix metalloproteinase 9 (MMP-9; assay: Human MMP-9 Immunoassay, R\&D Systems GmbH, Wiesbaden, Germany), tissue inhibitor of metalloproteinase 1 (TIMP-1, assay: Human TIMP-1 Immunoassay, R\&D Systems GmbH, Wiesbaden, Germany), B domain containing tenascin- $\mathrm{C}\left(\mathrm{B}^{+} \mathrm{Tn}-\right.$ C, assay: Tenascin-B Large (FNIII-B) ELISA, IBL International GmbH, Hamburg, Germany), endothelin 1 (ET-1, assay: Endothelin-1 Immunoassay, R\&D Systems GmbH, Wiesbaden, Germany) and neutrophil gelatinase-associated lipocalin (NGAL, assay: Human Lipocalin-2/NGAL Immunoassay, R\&D Systems, Wiesbaden, Germany). For 
the serum quantification of extra domain A containing fibronectin (ED-A $\left.{ }^{+} \mathrm{Fn}\right)$ and extra domain B containing fibronectin and $\left(\mathrm{ED}^{-\mathrm{B}^{+}} \mathrm{Fn}\right)$, we applied protocols, which were recently established and validated in our group [22, $32,33]$ because commercial ELISA assays are not available at present.

\subsection{Statistical analyses}

Statistical analyses were performed using IBM SPSS statistical software, version 25.0 (IBM SPSS Statistics for Windows. Armonk, NY, USA). Data are expressed as mean/median \pm standard deviation, as appropriate. MannWhitney-U test was used to test for significant differences between the groups with respect to clinical, laboratory, functional and imaging parameters as well as biomarker levels.

For the identification of independent predictors for one- and two-year mortality, multivariate regression analysis was performed by using a binary logistic model (backward elimination method: Wald). One- or two-year mortality was defined as the dependent variable.

In the identification cohort, $(\mathrm{n}=122)$, a wide range of baseline characteristics were assigned to three clusters with 4 to 10 items each. For each of the three clusters, a multivariate analysis was performed by using one-year mortality as dependent variable and the following items as cofounders. For cluster 1 (classical clinical parameters/comorbidities), New York Heart Association (NYHA) functional class, coronary artery disease (CAD), peripheral artery disease (PAD), diabetes mellitus (DM), chronic obstructive pulmonary disease (COPD), atrial fibrillation (Afib), presence of pacemaker (PM) and glomerular filtration rate (GFR) were included as cofounders. The items for cluster 2 (risk assessment scores) were EQ-VAS (0 to 100), CFS (1 to 9), STS score (\%) and staging classification of extra-valvular cardiac damage (stage 0 to 4 ) and for cluster 3 (novel biomarkers of cardiovascular remodeling) MMP9, TIMP-1, B ${ }^{+}$Tn-C, ET-1, NGAL, ED-A ${ }^{+}$Fn, ED-B ${ }^{+}$Fn and BNP serum levels.

In each cluster, one parameter could be identified to significantly increase one-year mortality risk in the identification cohort (Afib, CFS and TIMP-1). Basing on these findings, a simplified sum-score system was defined as follows: presence of Afib $($ no $=0$, yes $=1)$; dichotomized CFS $(1-4=0 ; 5-9=1)$; TIMP-1 range (cut-off value: 187.2 $\mathrm{ng} / \mathrm{mL}$; below $=0$, above $=1$ ). The resulting sum-score, denominated as Jena Mortality Score (JMS), ranged from level 0 to 3 . The one-year mortality rates per JMS level were calculated in the identification cohort and a multivariate analysis was performed as described including all parameters showing a $p$-value $<0.1$ in group comparison (survivors versus non-survivors after one year) except the 3 parameters contributing to JMS.

In a next step, to confirm our findings in a larger patients' collective, JMS was tested with respect to its pre- dictive value for one- and two-year mortality in the validation cohort $(\mathrm{n}=295)$. Therefore, survivors and nonsurvivors after one and two years were compared with respect to a wide range of clinical, laboratory, functional and imaging parameters including TIMP-1 serum levels using the Mann-Whitney- $U$ test. Then, multivariate regression analysis was performed, again by using a binary logistic model (backward elimination method: Wald), with oneor two-year mortality as dependent variable and all parameters showing a $p$-value $<0.1$ in group comparison (and not contributing to the included scores or classifications) as cofounders to identify independent predictors of long-term mortality.

Finally, Kaplan-Meier survival analysis including logrank test as well as receiver operating characteristic (ROC) analysis was performed to verify differences between high and low-risk patients according to JMS compared with STS score with respect to both, one- and two-year mortality. Comparison of area under the curve (AUC) values was performed by DeLong method using MedCalc statistical software (Version 20.023; MedCalc Software Ltd, Ostend, Belgium).

\section{Results}

\subsection{Identification cohort}

Baseline characteristics of the identification cohort, which represented a typical TAVI collective with a mean STS score of $4.8 \%$, are given in Table 1 . The one-year mortality rate was $14 \%$. By performing binary logistic regression analysis with one-year mortality as dependent variable and the items per cluster as cofounders as described in the material and methods section, Afib (cluster 1; OR 7.583, 95\% CI: 2.051-28.040, $p=0.002$ ), CFS (cluster 2; OR 2.258, 95\% CI: $1.262-4.039, p=0.006)$ and TIMP1 (cluster 3; OR 1.006, 95\% CI: 1.001-1.011, $p=0.019$ ) could be identified to significantly increase long-term mortality risk. These 3 parameters were integrated into a simplified sum-score (JMS) as follows as described. As cut-off value for TIMP-1, the median of the 122 patients, which was $187.2 \mathrm{ng} / \mathrm{mL}$, was chosen. The resulting JMS value ranged from 0 to 3 . The mortality rates per score level in the identification group were $0 \%$ for JMS $0,8.8 \%$ for JMS $1,22.2 \%$ for JMS 2 and $33.3 \%$ for JMS 3. When performing binary logistic regression analysis including all parameters showing a $p<0.1$ in group comparison (survivors versus non-survivors), except the 3 parameters contributing to the score, only JMS was independently associated with an increased risk for long-term mortality after TAVI (OR 2.720, 95\% CI: $1.467-5.042, p=0.001)$.

With respect to the biomarkers of cardiovascular remodeling measured by ELISA and included in cluster 3 as described in Material and Methods, the median values \pm standard deviation (SD) both, in the whole identification cohort $(\mathrm{n}=122)$ as well as in survivors $(\mathrm{n}=105)$ and nonsurvivors $(n=17)$ are given in Table 2. 
Table 1. Baseline characteristics of the identification compared to the validation cohort and corresponding $p$-values after group comparison.

\begin{tabular}{lccc}
\hline Parameter & Identification cohort $(\mathrm{n}=122)$ & Validation cohort $(\mathrm{n}=295)$ & $p$-value \\
\hline Age (years; mean \pm SD) & $78.2 \pm 8.0$ & $78.7 \pm 7.4$ & 0.829 \\
Female (\%) & 54.1 & 55.3 & 0.534 \\
STS (\%) & $4.8 \pm 2.8$ & $4.8 \pm 3.4$ & 1.000 \\
NYHA >II (\%) & 64.8 & 73.6 & 0.024 \\
CAD (\%) & 61.5 & 60.4 & 0.840 \\
PAD (\%) & 10.7 & 12.0 & 0.700 \\
Diabetes (\%) & 45.1 & 49.2 & 0.816 \\
COPD (\%) & 25.4 & 28.3 & 0.545 \\
Afib (\%) & 44.3 & 48.6 & 0.418 \\
PM (\%) & 16.4 & 15.8 & 0.882 \\
GFR (mL/min; mean \pm SD) & $52.1 \pm 22.5$ & $52.6 \pm 22.4$ & 0.883 \\
BNP (pg/mL; mean \pm SD) & $863.5 \pm 1379.3$ & $913.9 \pm 1747.9$ & 0.861 \\
LVEF (\%; mean \pm SD) & $55.7 \pm 14.0$ & $57.6 \pm 14.9$ & 0.136 \\
AS subtype (\%) & & & 0.760 \\
HGAS & 73.8 & 71.8 & \\
LGAS & 14.8 & 17.8 & \\
PLFLGAS & 11.5 & 10.5 & \\
Stage 0-4 (\%) & & 3.1 & \\
0 & 3.3 & 12.6 & \\
1 & 18.2 & 50.9 & \\
2 & 49.6 & 21.5 & \\
3 & 19.0 & 11.9 & \\
4 & 9.9 & 3.9 & \\
\hline STS & & & \\
\hline
\end{tabular}

STS score, Society of Thoracic Surgeons score; NYHA, New York Heart Association; CAD, coronary artery disease; PAD, peripheral artery disease; COPD, chronic obstructive pulmonary disease; Afib, atrial fibrillation; PM, pace maker; GFR, glomerular filtration rate; BNP, brain natriuretic peptide; LVEF, left ventricular ejection fraction; AS, aortic stenosis; HGAS, high gradient aortic stenosis; LGAS, low gradient aortic stenosis; PLFLGAS paradoxical low flow low gradient aortic stenosis; stage, stages of extra-valvular cardiac damage according.

\subsection{Validation cohort}

Baseline characteristics of the validation cohort, which again represented a typical TAVI collective with a mean STS score of $4.8 \%$, are given in Table 1 . The mortality rate was $18 \%$ after one and $23 \%$ after two years.

Binary logistic regression analysis with one- and twoyear mortality as dependent variable and STS score (STS), staging of extra-valvular cardiac damage (stage), presence of high gradient aortic stenosis (HGAS), brain natriuretic peptide (BNP), EQ visual analogue scale (EQ-VAS) and JMS as cofounders was performed. The cofounders were defined as all parameters not contributing to the implemented scores and showing a $p$-value $<0.1$ in group comparison between survivors and non-survivors after one year.

As a result, besides STS score, only JMS could be proven to serve as independent predictor of both, one-year (JMS: OR 1.626, 95\% CI: 1.053-2.509, $p=0.028$; STS score: OR $1.180,95 \%$ CI: $1.052-1.325, p=0.005)$ and two- year (JMS: OR 1.649, 95\% CI: 1.093-2.488, $p=0.017$; STS score: OR 1.253, 95\% CI: $1.106-1.419, p<0.001)$ mortality.

The one-/two-year mortality rates per score level in the validation cohort were $1.75 / 2.62 \%$ for JMS $0,3.93 / 4.80 \%$ for JMS 1, 6.11/7.86\% for JMS 2 and $6.55 / 7.86 \%$ for JMS 3.

After dichotomization of patients into a low- and highrisk group according to JMS and STS score (JMS: 0 or $1=$ low-risk, 2 or $3=$ high-risk; STS score: $<4 \%=$ low-risk, $>4 \%=$ high-risk) Kaplan-Meier survival analysis was performed. The results are shown in Fig. 1a (JMS) and Fig. 1b (STS score). In brief, Kaplan-Meier survival analysis displayed a significant survival benefit for the low-risk groups according to both, JMS and STS score, after one and two years ( $p<0.05$ for all). Of note, survival rate in the JMS high risk group is lower $(66.0 \%)$ compared to those in the STS score high risk group (71.4\%). ROC analysis for the discrimination of survivors from non-survivors revealed an 
Table 2. Detailed results of biomarker measurements performed in the study (median values \pm standard deviation [SD]).

\begin{tabular}{lcccc}
\hline \multirow{2}{*}{ Biomarker } & Identification cohort & Survivors & Non-survivors & $p$ value \\
\cline { 2 - 5 } & $(\mathrm{n}=122)$ & after 1 year $(\mathrm{n}=105)$ & after 1 year $(\mathrm{n}=17)$ & (survivors vs. non-surviors) \\
\hline MMP-9 $(\mathrm{ng} / \mathrm{mL})$ & $400 \pm 339$ & $405 \pm 341$ & $369 \pm 331$ & 0.618 \\
TIMP-1 $(\mathrm{ng} / \mathrm{mL})$ & $187 \pm 94$ & $182 \pm 83$ & $287 \pm 132$ & 0.053 \\
$\mathrm{~B}^{+}$Tn-C $(\mathrm{ng} / \mathrm{mL})$ & $784 \pm 726$ & $755 \pm 680$ & $920 \pm 971$ & 0.656 \\
ET-1 $(\mathrm{ng} / \mathrm{mL})$ & $2.5 \pm 1.3$ & $2.5 \pm 1.3$ & $3.2 \pm 1.4$ & 0.190 \\
NGAL $(\mathrm{ng} / \mathrm{mL})$ & $137 \pm 166$ & $137 \pm 142$ & $140 \pm 269$ & 0.819 \\
ED-A $^{+} \mathrm{Fn}(\mu \mathrm{g} / \mathrm{mL})$ & $10.5 \pm 12.0$ & $10.7 \pm 12.2$ & $9.6 \pm 10.5$ & 0.399 \\
ED-B $^{+}$Fn $(\mu \mathrm{g} / \mathrm{mL})$ & $4.1 \pm 3.2$ & $4.3 \pm 3.2$ & $2.9 \pm 2.5$ & 0.482 \\
\hline
\end{tabular}

Jena Mortality Score (JMS)

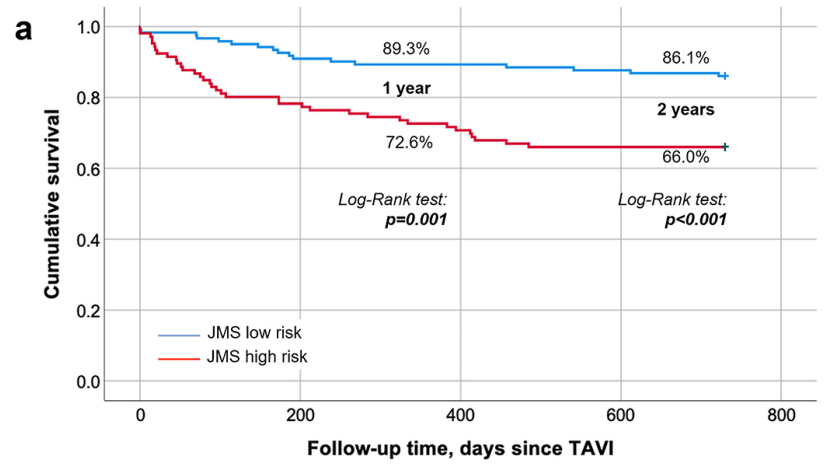

Society of Thoracic Surgeons (STS) score

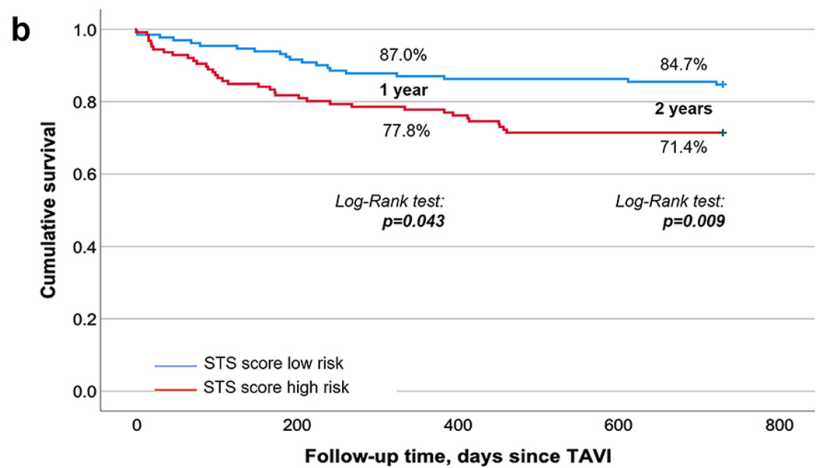

Fig. 1. Kaplan-Meier survival analysis for JMS and STS scoredisplaying a significant survival benefit for the low-risk groups according after one and two years. (a) JMS. (b) STS score.

AUC for JMS of 0.676 and for the STS score of 0.710 after one year (Fig. 2a,b, $p=0.534$ ) and an AUC for JMS of 0.686 and for the STS score of 0.725 after two years (Fig. 2c,d, $p$ $=0.437)$.

\subsection{Tissue inhibitor of metalloproteinase 1 (TIMP-1) as novel prognostic biomarker in TAVI patients}

With explicit focus on the serum levels of TIMP-1, which have been already reported above as part of JMS, the following observations are of value to be reported separately. When comparing survivors and non-survivors after one and two years, there are significantly increased TIMP1 concentrations in the non-survivors $(p<0.001$ for both timepoints) (Fig. 3a,b). ROC analysis revealed an AUC of 0.678 for the discrimination of survivors and non-survivors after one and an AUC of 0.670 after two years, which are superior compared to, e.g., baseline BNP levels (AUC 0.642 after one and 0.639 after two years) or baseline GFR (AUC 0.587 after one and 0.586 after two years).

\section{Discussion}

The newly introduced Jena Mortality Score (JMS) for the prediction of long-term mortality after TAVI entails not only classical clinical, laboratory or imaging parameters but, for the first time also a novel biomarker of cardiovascular extracellular matrix accumulation (ECM) and fi- brosis. Thus, it represents an innovative approach possibly contributing to a more precise risk assessment facilitating patient-centered heart-team decision making as recommended by current guidelines [34]. Mortality prediction in TAVI patients is a remaining challenge, especially when focusing on long-term outcomes. This situation is due to the fact that most available scores were developed and validated in SAVR patients to predict short-term, e.g., 30-day, mortality. Therefore, their use in TAVI patients leads to an overestimation of 30-day mortality, at least in the elective setting $[8,9,35]$. Although, several TAVI specific scores have been proposed in recent years, their additive value in daily clinical practice is limited since most scores focus on 30-day mortality and underperform in external validation cohorts $[13,15,36]$. Especially for the prediction of longterm mortality, only small cohorts have been the objects of recent studies. While there were some efforts to develop TAVI risk prediction models for one-year mortality, there are no data for longer survival periods, e.g., two years after TAVI.

Despite the availability of various studies investigating the value of novel biomarkers of cardiovascular remodeling for improving the diagnosis or estimating the prognosis of AS patients [22,25,26,37], to our best knowledge, none of these markers has ever been implemented into a long-term mortality risk prediction model after TAVI. Thus, 
ROC anaylsis

(survivors vs. non-survivors after 1 year)

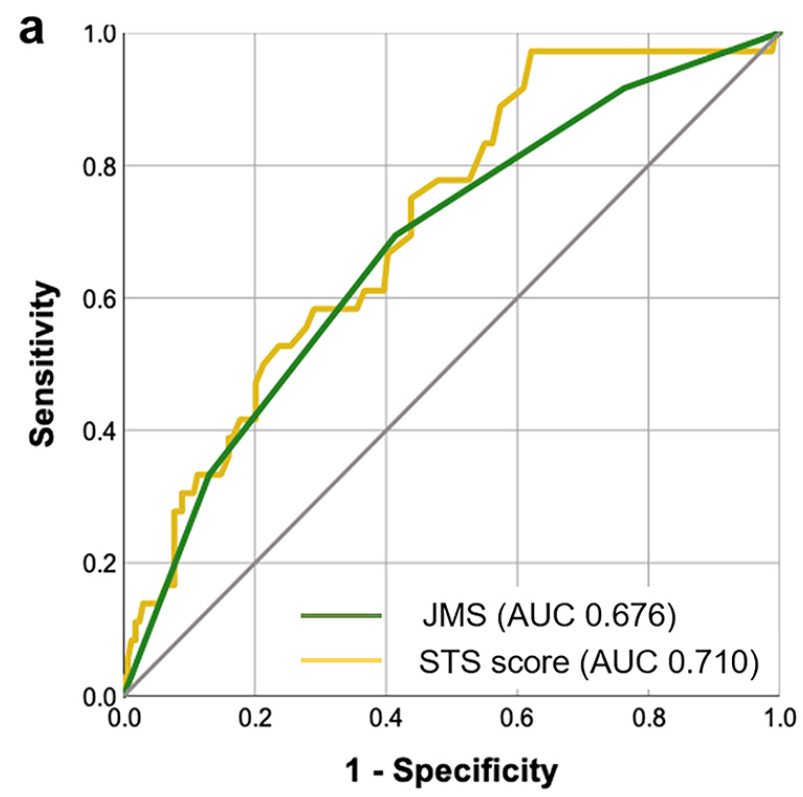

ROC anaylsis

(survivors vs. non-survivors after 2 years)

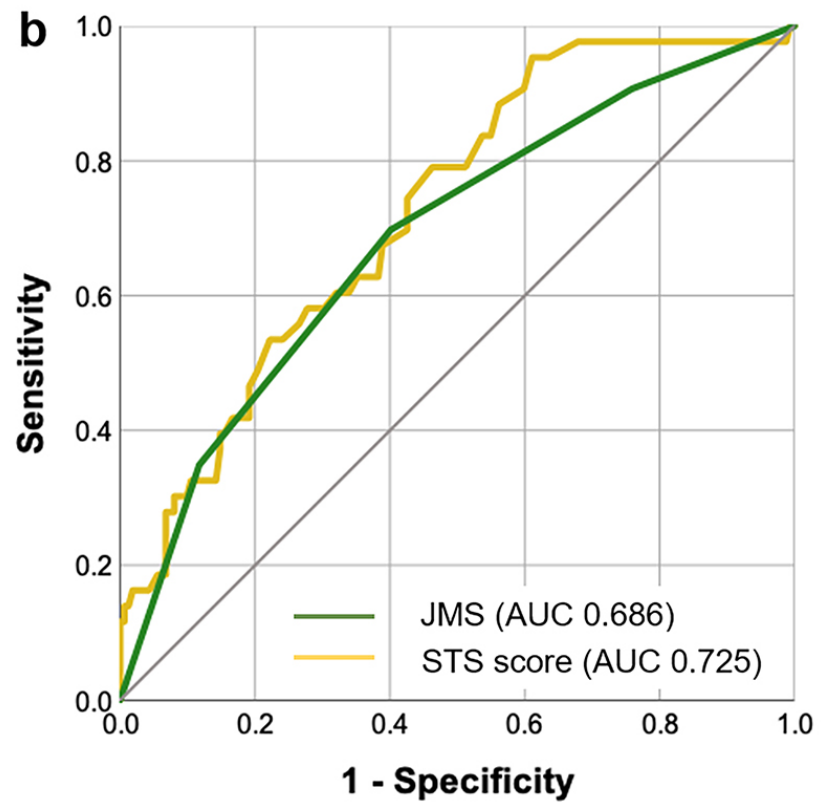

Fig. 2. ROC analysis for the discrimination of survivors from non-survivors revealing an AUC for the JMS of 0.676 and for the STS score of 0.710 after one year and an AUC for the JMS of $\mathbf{0 . 6 8 6}$ and for the STS score of 0.725 after two years. (a) and (b) results after one year.
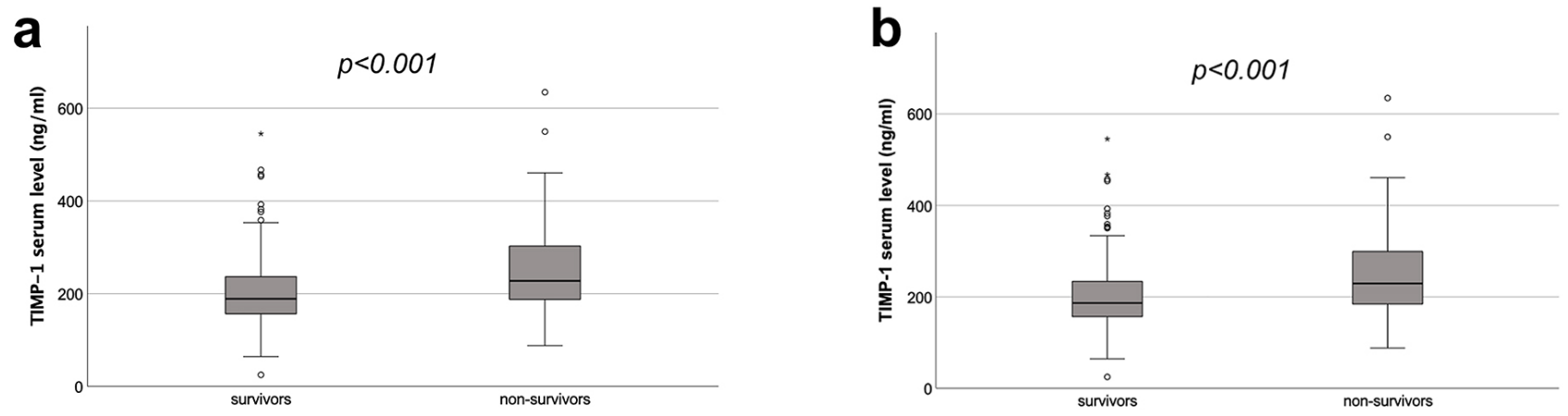

Fig. 3. Baseline TIMP-1 concentrations in survivors and non-survivors after one and two years showing significantly increased levels in non-survivors for both time-points. (a) Results after one year. (b) After two years.

the JMS presented here, might fill this gap by integrating circulating levels of TIMP-1, which is a well-known biomarker of cardiovascular ECM accumulation and fibrosis [22,38-40].

When interpreting the results of the current study, it has to be clearly mentioned that the number of patients included in both, the identification as well as the validation cohort, is limited and that we cannot provide external validation since both cohorts were derived from the Jenaer Aortenklappenregister (JAKR), which includes patients from only one center.

Nevertheless, when comparing JMS versus STS score derived low- versus high-risk patients with respect to oneand two-year survival, JMS derived risk class performed better in our study. This goes in line with recent reports on the limited value of STS score-based risk assessment in TAVI populations, especially with respect to long-term outcomes [10].

Interestingly, a very recent meta-regression analysis of 8 randomized trials investigating TAVI in high-, intermediate- and low-risk patients, impressively demonstrated that there was no association between STS score and hazard ratio for two-year mortality and thereby questions the usefulness of surgical risk-scoring in TAVI patients in general [41].

In contrast to most studies investigating TAVI specific risk assessment models, which aimed to predict one-year mortality $[16,17,42]$, JMS, described by us, is even capa- 
ble to predict two-year mortality. However, its validity in larger external cohorts has still to be proven, which represents a clear limitation of our study. Of note, to overcome this issue will remain challenging since TIMP-1 serum levels are not part of routine laboratory diagnostics and not available for retrospective analyses. On the other hand, the development of a TIMP-1 point-of-care test should be very easy to realize since it is already available for a variety of other cardiac biomarkers, e.g., Troponin T (TnT), brain natriuretic peptide (BNP), D-dimer or even novel markers like heart-type fatty acid-binding protein (H-FABP) [43]. Once available, assessment of JMS would be not only very simple but could also be performed extremely fast at bedside. This might represent a great advantage compared to nearly all the other available scores, e.g., the complex STS score, which takes, depending on the physician's experience, at least several minutes. However, it will take certain efforts in the future to somehow implement TIMP-1 measurement in clinical routine for risk assessment of AS patients.

Besides TIMP-1, JMS also implies the presence of Afib and the CFS, which are both very well-known mortality predictors in TAVI patients $[10,44]$. In that context, especially frailty assessment scores for TAVI risk evaluation facilitating heart-team decision making have been recommended frequently in the literature [45].

Moreover, a great advantage of JMS is the fact that it has been developed in TAVI patients. In contrast, the STS score, which has been widely used for risk assessment in TAVI patients over the last decade, is derived from SAVR patients [12]. Against that background, we would like to suggest integrating both scores in risk assessment during heart team evaluation, especially in patients between 70 and 75 years, in which current ESC guidelines place special emphasis on individualized decision making between SAVR and TAVI [34].

Taken together, we suggest a simple and fast to perform risk-prediction model to estimate long-term mortality after transfemoral TAVI performed in the elective setting. Although, external validation remains a mandatory future task, we would like to place special emphasis on the incorporation of novel biomarkers of cardiovascular tissue remodeling into TAVI risk assessment scores.

\section{Conclusions}

JMS, including TIMP-1 as a novel biomarker of cardiac extracellular matrix accumulation and fibrosis, could serve as a simple tool to assess long-term mortality risk after TAVI and might thereby contribute to a more precise stratification of individual risk. A great advantage is its simplicity and, compared to many other scores, extremely short performance time.

\section{Author contributions}

LB and MF designed the research study. LB, KG, MD, SMW, PCS and MF performed the research. LB, KG,
$\mathrm{CJ}$ and MF provided help and advice on the ELISA experiments. LB, MF, AP, CJ and PCS analyzed the data. All authors contributed to editorial changes in the manuscript. All authors read and approved the final manuscript.

\section{Ethics approval and consent to participate}

All patients included gave written informed consent for participation and the local ethics committee of the University Hospital Jena has approved the study (registration number: 4815-06/16).

\section{Acknowledgment}

The authors would like to thank Annett Schmidt for excellent technical assistance.

\section{Funding}

This work was supported by funding from the Foundation "Else Kröner-Fresenius-Stiftung" within the Research Program "Else Kröner-Forschungskolleg AntiAge".

\section{Conflict of interest}

The authors declare no conflict of interest.

\section{References}

[1] Leon MB, Smith CR, Mack MJ, Makkar RR, Svensson LG, Kodali SK, et al. Transcatheter or Surgical Aortic-Valve Replacement in Intermediate-Risk Patients. New England Journal of Medicine. 2016; 374: 1609-1620.

[2] Mack MJ, Leon MB, Thourani VH, Makkar R, Kodali SK, Russo M, et al. Transcatheter Aortic-Valve Replacement with a Balloon-Expandable Valve in Low-Risk Patients. the New England Journal of Medicine. 2019; 380: 1695-1705.

[3] Makkar RR, Fontana GP, Jilaihawi H, Kapadia S, Pichard AD, Douglas PS, et al. Transcatheter aortic-valve replacement for inoperable severe aortic stenosis. The New England Journal of Medicine. 2012; 366: 1696-1704.

[4] Popma JJ, Deeb GM, Yakubov SJ, Mumtaz M, Gada H, O’Hair D, et al. Transcatheter Aortic-Valve Replacement with a SelfExpanding Valve in Low-Risk Patients. The New England Journal of Medicine. 2019; 380: 1706-1715.

[5] Smith CR, Leon MB, Mack MJ, Miller DC, Moses JW, Svensson LG, et al. Transcatheter versus surgical aortic-valve replacement in high-risk patients. The New England Journal of Medicine. 2011; 364: 2187-2198.

[6] Baumgartner H. The 2017 ESC/EACTS guidelines on the management of valvular heart disease : what is new and what has changed compared to the 2012 guidelines? Wiener Klinische Wochenschrift. 2018; 130: 168-171.

[7] Nishimura RA, Otto CM, Bonow RO, Carabello BA, Erwin JP, Fleisher LA, et al. 2017 AHA/ACC Focused Update of the 2014 AHA/ACC Guideline for the Management of Patients with Valvular Heart Disease: a Report of the American College of Cardiology/American Heart Association Task Force on Clinical Practice Guidelines. Journal of the American College of Cardiology. 2017; 70: 252-289.

[8] Martin GP, Sperrin M, Ludman PF, de Belder MA, Gale CP, Toff WD, et al. Inadequacy of existing clinical prediction models for predicting mortality after transcatheter aortic valve implantation. American Heart Journal. 2017; 184: 97-105.

[9] Wang TKM, Wang MTM, Gamble GD, Webster M, Ruygrok 
PN. Performance of contemporary surgical risk scores for transcatheter aortic valve implantation: a meta-analysis. International Journal of Cardiology. 2017; 236: 350-355.

[10] Gupta T, Joseph DT, Goel SS, Kleiman NS. Predicting and measuring mortality risk after transcatheter aortic valve replacement. Expert Review of Cardiovascular Therapy. 2021; 19: 247-260.

[11] Nashef SAM, Roques F, Sharples LD, Nilsson J, Smith C, Goldstone AR, et al. EuroSCORE II. European Journal of CardioThoracic Surgery. 2012; 41: 734-735.

[12] O'Brien SM, Shahian DM, Filardo G, Ferraris VA, Haan CK, Rich JB, et al. The Society of Thoracic Surgeons 2008 cardiac surgery risk models: part 2-isolated valve surgery. The Annals of Thoracic Surgery. 2009; 88: S23-S42.

[13] Capodanno D, Barbanti M, Tamburino C, D'Errigo P, Ranucci $\mathrm{M}$, Santoro G, et al. A simple risk tool (the OBSERVANT score) for prediction of 30-day mortality after transcatheter aortic valve replacement. The American Journal of Cardiology. 2014; 113: $1851-1858$

[14] D’Ascenzo F, Capodanno D, Tarantini G, Nijhoff F, Ciuca C, Rossi ML, et al. Usefulness and validation of the survival posT TAVI score for survival after transcatheter aortic valve implantation for aortic stenosis. The American Journal of Cardiology. 2014; 114: 1867-1874.

[15] Iung B, Laouénan C, Himbert D, Eltchaninoff H, Chevreul K, Donzeau-Gouge P, et al. Predictive factors of early mortality after transcatheter aortic valve implantation: individual risk assessment using a simple score. Heart. 2014; 100: 1016-1023.

[16] Debonnaire P, Fusini L, Wolterbeek R, Kamperidis V, van Rosendael P, van der Kley F, et al. Value of the "TAVI2-SCORe" Versus Surgical Risk Scores for Prediction of one Year Mortality in 511 Patients who Underwent Transcatheter Aortic Valve Implantation. The American Journal of Cardiology. 2015; 115: 234-242.

[17] Hermiller JB, Yakubov SJ, Reardon MJ, Deeb GM, Adams DH, Afilalo J, et al. Predicting Early and Late Mortality after Transcatheter Aortic Valve Replacement. Journal of the American College of Cardiology. 2016; 68: 343-352.

[18] Lopez-Marco A, Miller H, Youhana A, Ashraf S, Zaidi A, Bhatti $\mathrm{F}$, et al. Low-flow low-gradient aortic stenosis: surgical outcomes and mid-term results after isolated aortic valve replacement. European Journal of Cardio-Thoracic Surgery. 2016; 49: $1685-1690$.

[19] Salaun E, Clavel MA, Hahn RT, Jaber WA, Asch FM, Rodriguez $\mathrm{L}$, et al. Outcome of Flow-Gradient Patterns of Aortic Stenosis After Aortic Valve Replacement: An Analysis of the PARTNER 2 Trial and Registry. Circulation: Cardiovascular Interventions. 2020; 13: e008792.

[20] Alushi B, Beckhoff F, Leistner D, Franz M, Reinthaler M, Stähli $\mathrm{BE}$, et al. Pulmonary Hypertension in Patients with Severe Aortic Stenosis: Prognostic Impact after Transcatheter Aortic Valve Replacement. JACC: Cardiovascular Imaging. 2019; 12: 591601.

[21] Généreux P, Pibarot P, Redfors B, Mack MJ, Makkar RR, Jaber WA, et al. Staging classification of aortic stenosis based on the extent of cardiac damage. European Heart Journal. 2017; 38: 3351-3358.

[22] Bäz L, Dannberg G, Grün K, Westphal J, Möbius-Winkler S, Jung C, et al. Serum Biomarkers of Cardiovascular Remodelling Reflect Extra-Valvular Cardiac Damage in Patients with Severe Aortic Stenosis. International Journal of Molecular Sciences. 2020; $21: 4174$.

[23] Li Y, Pei H, Zhou C, Lou Y. Pre-procedural elevated cardiac troponin predict risk of long-term all-cause mortality after tran- scatheter aortic valve replacement: a meta-analysis of prospective studies. Biomarkers. 2020; 25: 164-170.

[24] O'Leary JM, Clavel MA, Chen S, Goel K, O'Neill B, Elmariah $\mathrm{S}$, et al. Association of Natriuretic Peptide Levels After Transcatheter Aortic Valve Replacement With Subsequent Clinical Outcomes. JAMA Cardiology. 2020; 5: 1113-1123.

[25] Krau N, Lünstedt N, Freitag-Wolf S, Brehm D, Petzina R, Lutter $\mathrm{G}$, et al. Elevated growth differentiation factor 15 levels predict outcome in patients undergoing transcatheter aortic valve implantation. European Journal of Heart Failure. 2015; 17: $945-$ 955.

[26] Wernly B, Lichtenauer M, Jirak P, Eder S, Reiter C, Kammler J, et al. Soluble ST2 predicts 1-year outcome in patients undergoing transcatheter aortic valve implantation. European Journal of Clinical Investigation. 2017; 47: 149-157.

[27] Muessig JM, Lichtenauer M, Wernly B, Kelm M, Franz M, Bäz $\mathrm{L}$, et al. Insulin like growth factor binding protein 2 (IGFBP-2) for risk prediction in patients with severe aortic stenosis undergoing Transcatheter Aortic Valve Implantation (TAVI). International Journal of Cardiology. 2019; 277: 54-59.

[28] EuroQol Group. EuroQol-a new facility for the measurement of health-related quality of life. Health Policy. 1990. 16: 199-208.

[29] Bäz L, Wiesel M, Möbius-Winkler S, Westphal JG, Schulze PC, Franz M, et al. Depression and anxiety in elderly patients with severe symptomatic aortic stenosis persistently improves after transcatheter aortic valve replacement (TAVR). International Journal of Cardiology. 2020; 309: 48-54.

[30] Bäz L, Puscholt M, Lasch C, Diab M, Möbius-Winkler S, Schulze PC, et al. Delayed Improvement of Depression and Anxiety after Transcatheter Aortic Valve Implantation (TAVI) in Stages of Extended Extra-Valvular Cardiac Damage. Journal of Clinical Medicine. 2021; 10: 1579.

[31] Rockwood K, Song X, MacKnight C, Bergman H, Hogan DB, McDowell I, et al. A global clinical measure of fitness and frailty in elderly people. Canadian Medical Association Journal. 2005; 173: 489-495.

[32] Bäz L, Roßberg M, Grün K, Kretzschmar D, Berndt A, Schulze $\mathrm{PC}$, et al. Serum Liberation of Fetal Fibronectin Variants in Patients with Pulmonary Hypertension: ED-A(+) Fn as Promising Novel Biomarker of Pulmonary Vascular and Right Ventricular Myocardial Remodeling. Journal of Clinical Medicine. 2021; 10: 2559.

[33] Ziffels B, Ospel J, Grün K, Neri D, Pfeil A, Fritzenwanger M, et al. Detection of Soluble ED-a+Fibronectin and Evaluation as Novel Serum Biomarker for Cardiac Tissue Remodeling. Disease Markers. 2016; 2016: 1-11.

[34] Vahanian A, Beyersdorf F, Praz F, Milojevic M, Baldus S, Bauersachs J, et al. $2021 \mathrm{ESC} / \mathrm{EACTS}$ Guidelines for the management of valvular heart disease. European Heart Journal. 2021. (in press)

[35] Piazza N, Wenaweser P, van Gameren M, Pilgrim T, Tzikas A, Tsikas A, et al. Relationship between the logistic EuroSCORE and the Society of Thoracic Surgeons Predicted Risk of Mortality score in patients implanted with the CoreValve ReValving system-a Bern-Rotterdam Study. American Heart Journal. 2010; 159: 323-329.

[36] Schiller W, Barnewold L, Kazmaier T, Beckmann A, Masseli F, Welz A, et al. The German Aortic Valve Score II. European Journal of Cardio-Thoracic Surgery. 2017; 52: 881-887.

[37] Mirna M, Holnthoner M, Topf A, Jirak P, Fejzic D, Paar V, et $a l$. Tumor necrosis factor alpha - an underestimated risk predictor in patients undergoing transcatheter aortic valve replacement (TAVR)? Journal of Clinical Laboratory Analysis. 2021; 35: e23977. 
[38] Franz M, Berndt A, Altendorf-Hofmann A, Fiedler N, Richter $\mathrm{P}$, Schumm J, et al. Serum levels of large tenascin-C variants, matrix metalloproteinase- 9 , and tissue inhibitors of matrix metalloproteinases in concentric versus eccentric left ventricular hypertrophy. European Journal of Heart Failure. 2009; 11: 10571062.

[39] Franz M, Berndt A, Neri D, Galler K, Grün K, Porrmann $\mathrm{C}$, et al. Matrix metalloproteinase-9, tissue inhibitor of metalloproteinase- $1, \mathrm{~B}^{+}$tenascin-C and $\mathrm{ED}-\mathrm{a}^{+}$fibronectin in dilated cardiomyopathy: potential impact on disease progression and patients' prognosis. International Journal of Cardiology. 2013; 168: 5344-5351.

[40] Ben Braiek A, Chahed H, Dumont F, Abdelhak F, Hichem D, Gamra $\mathrm{H}$, et al. Identification of biomarker panels as predictors of severity in coronary artery disease. Journal of Cellular and Molecular Medicine. 2021; 25: 1518-1530.

[41] Baro R, Cura F, Belardi J, Brugaletta S, Lamelas P. Surgical Risk Scoring in TAVR: still Needed? A Metaregression Analy- sis. Current Problems in Cardiology. 2021; 46: 100875.

[42] Seiffert M, Sinning J, Meyer A, Wilde S, Conradi L, VasaNicotera M, et al. Development of a risk score for outcome after transcatheter aortic valve implantation. Clinical Research in Cardiology. 2014; 103: 631-640.

[43] Schols AMR, Stakenborg JPG, Dinant G, Willemsen RTA, Cals JWL. Point-of-care testing in primary care patients with acute cardiopulmonary symptoms: a systematic review. Family Practice. 2018; 35: 4-12.

[44] Yamamoto M, Otsuka T, Shimura T, Yamaguchi R, Adachi Y, Kagase A, et al. Clinical risk model for predicting 1-year mortality after transcatheter aortic valve replacement. Catheterization and Cardiovascular Interventions. 2021; 97

[45] Schoenenberger AW, Moser A, Bertschi D, Wenaweser P, Windecker S, Carrel T, et al. Improvement of Risk Prediction after Transcatheter Aortic Valve Replacement by Combining Frailty with Conventional Risk Scores. JACC: Cardiovascular Interventions. 2018; 11: 395-403. 